\title{
Teaching Electromagnetic Interference on Microcontrollers through Lab Experiments
}

\author{
http://dx.doi.org/10.3991/ijep.v3i2.2252 \\ Chiu H. Choi \\ University of North Florida, Jacksonville, FL, USA
}

\begin{abstract}
New lab experiments were developed for the students to investigate the effect of electromagnetic interference on the operation of microcontrollers. The objectives of the experiments include exposing students to the effect of noisy power supply on microcontroller performance and to learn how to use modern instruments for recording the results. In the experiments, the noise is restricted to electromagnetic interference from external sources at radio frequencies picked up by the power supply conductors. As wireless communication technologies have been widely used, such lab investigation is of current interest to the students. There was no simple way to produce the radio frequency interference of sufficient magnitude in our lab. A method was used to emulate the picking up of such interference on the power supply conductors. This paper describes such a method. It also describes the new experiments, their benefits for the students, the evaluations, and suggestions for improvements.
\end{abstract}

Index Terms - engineering pedagogy, electromagnetic interference, microcontroller applications.

\section{INTRODUCTION}

Microcontrollers are ubiquitous. They are found in automobiles, cell phones, appliances, and many wireless products. In applications such as automobiles, tens of microcontrollers can be found in the electronic networks of a single vehicle. The power supply conductors of these microcontrollers are frequently subjected to various electromagnetic interferences coming from the engines, alternators and motors. To develop lab experiments of electromagnetic interference on microcontroller operation is of current interest to the students. Such lab experiments have been developed and incorporated into an embedded control systems course and an electromagnetics course. The embedded control systems course is one of the graduate courses in our MSEE curriculum. It is a hands-on course teaching students how to design proportional, proportional-derivative, and other controllers and to implement these controllers into a microcontroller such as Freescale (formerly Motorola) 16-bit microcontrollers. Typical enrollment of this course is about seven to ten graduate students.

The electromagnetics course is an undergraduate electrical engineering course that must be taken by our junior or senior electrical engineering students. The enrollment is typically thirty to forty students. The syllabus of the electromagnetics course covers vector calculus, electro- statics, magnetostatics, Maxwell equations, plane wave propagation, transmission lines and electromagnetic interference. The course was theoretical before and has been balanced with the hands-on lab experiments described in this paper.

In the literature we find a variety of microcontroller labs offered in engineering programs. For instance, in [1], it describes a remote microcontroller lab based on an 80C537. The paper presents a new way to interact such microcontroller system through the internet. In [2] the paper describes some cooperative experiential learning activities for improving embedded systems skills. The experiential learning activities are constructing and troubleshooting of a microcontroller-based project. In [3], the paper proposes teaching the microcontroller as a powerful design tool in solving various industrial monitoring and control problems. In [4], this paper describes a curriculum that addresses the needs of embedded systems and the effort of delivering learning modules that supplement traditional courses. In [5] the paper describes the development of microcontroller and FPGA-based course materials. None of the literature above covers the topic of electromagnetic interference on microcontroller operations through lab experiments.

Further searching of lab experience for electromagnetics courses, e.g., [6], [7], and [8], indicates that the coverage of electromagnetic interference on microcontrollers is rarely available. Such a gap has been identified. The purpose of this paper is to fill this gap.

This paper describes new lab experiments for investigating the effects of electromagnetic interference on the operation of microcontrollers. The electromagnetic interference is restricted to radio frequency (RF) interference picked up by the power supply conductors from external sources such as engines, large electric motors, and wireless communication networks. Students find these labs relevant because microcontrollers are incorporated in many applications and the interference sources are familiar to them. There is no simple way to produce the radio frequency interference of sufficient magnitude to be picked up by the power supply conductors in our lab. A method was used to emulate the picking up of such interference by the power supply conductors.

The rest of this paper is organized as follows: the objectives of the lab experiments are provided in the rest of this section. The next section describes the method for emulating the picking up of the electromagnetic interferences. Section III describes the details of the experiments with experimental results. Section IV provides the evaluation results in regard to the objectives of the 
experiments. Concluding remarks are provided in Section $\mathrm{V}$.

The objectives of the lab experiments include investigating the effect of radio frequency interference on the operation of microcontrollers, enhancing the hands-on skills of the students, teaching the students how to operate sophisticated instruments such as mixed signal oscilloscopes and radio frequency signal generators, and the use of circuit simulation software for determining the effectiveness of the emulation method. Some of the lab experiments were open-ended and the interested students can conduct further investigation on their own.

\section{EMULATING ELECTROMAGNETIC INTERFERENCES}

There was no simple way to produce the radio frequency interference of sufficient magnitude in our lab. A method was used to emulate the picking up of such interference on the power supply conductors. A superposition circuit in Fig. 1 was used for emulating the radio frequency interference. Such circuit can be found in procedures for testing speed measurement devices such as in [9]. This circuit superimposes two sources together. The first source is the $5 \mathrm{~V} \mathrm{DC}$ input produced by a DC power supply. The second source is the RF signals in Fig. 1. The radio frequency input was generated by a HP 8656B Signal Generator [10]. The signal generator can generate both FM and AM signals in the frequency range of $100 \mathrm{KHz}$ to $990 \mathrm{MHz}$. The output of the superposition circuit in Fig. 1 was connected to the power and ground pins of a microcontroller.

The superposition is explained as follows: The $1 \mathrm{uF}$ capacitor behaves like an open circuit to the $5 \mathrm{~V}$ DC source. The $5 \mathrm{mH}$ inductor behaves like a short circuit to the $5 \mathrm{~V}$ DC source. Therefore the $5 \mathrm{~V}$ DC input can emerge at the output of the circuit. The RF signal source can pass through the $0.1 \mathrm{uF}$ because a capacitor presents small impedance to high frequency signals. The $5 \mathrm{mH}$ inductor can block the RF signals from going into the left half of the circuit because it presents very high impedance to the RF signals. Therefore the RF signal will also emerge at the output of the superposition circuit without leaking through the inductor. The output of the superposition circuit is to be connected to the power supply pins of a microcontroller in the experiments. The microcontroller received noisy power from this superposition circuit and the noise is at radio frequency.

Transient analysis of the superposition circuit in Fig. 1 was performed using a cloud-based circuit simulation software CircuitLab [11] available on the internet. The RF signal in Fig. 1 was of the form $0.5 \sin (\omega t)$, where $\omega=2 \pi \mathrm{f}$ and $f=10 \mathrm{MHz}$. The output signal in Fig. 1 is plotted in Fig. 2. As the graph in Fig. 2 indicated, the output signal is the superposition of the $5 \mathrm{~V} \mathrm{DC}$ input and the RF signal $0.5 \sin (\omega t)$. This confirms that the superposition circuit in Fig. 1 is performing the superposition of the DC input and the RF signal. RF signal of other frequencies were simulated also and consistent superposition results were obtained.

\section{LAB EXPERIMENTS AND RESULTS}

Students investigated in the lab experiments the effect of noisy power supply on microcontroller operation. The noise was at radio frequency and was superimposed on top of the $5 \mathrm{~V}$ DC signal through the superposition cir-

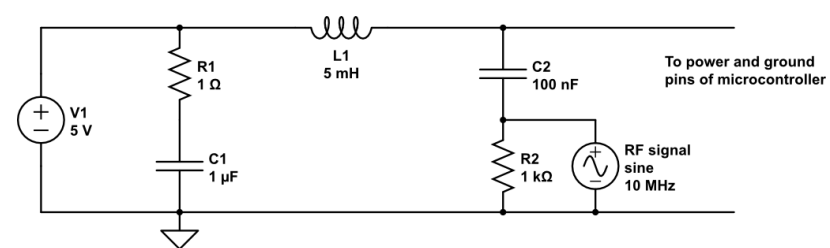

Figure 1. superposition circuit

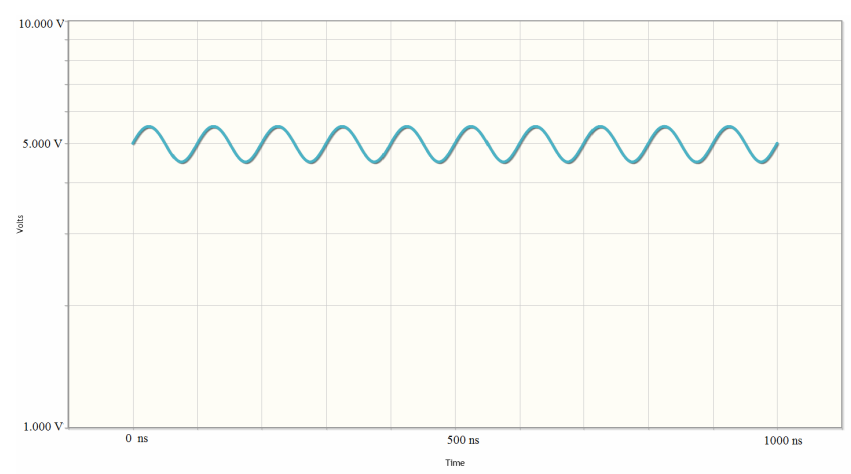

Figure 2. Output of the superposition circuit

cuit. Various microcontrollers were tested by the students, for example, Freescale Semiconductors' MC9S12C32 16 bit microcontroller [12] and Microchip's PIC 16F690 microcontroller [13].

\section{A. The first experiment}

In the first experiment, the students investigated the effect of the noisy power supply on a four-bit binary upcounter implemented as a $\mathrm{C}$ program running on a Freescale MC9S12C32 microcontroller. The four-bit binary up- counter program is shown in Fig. 3.

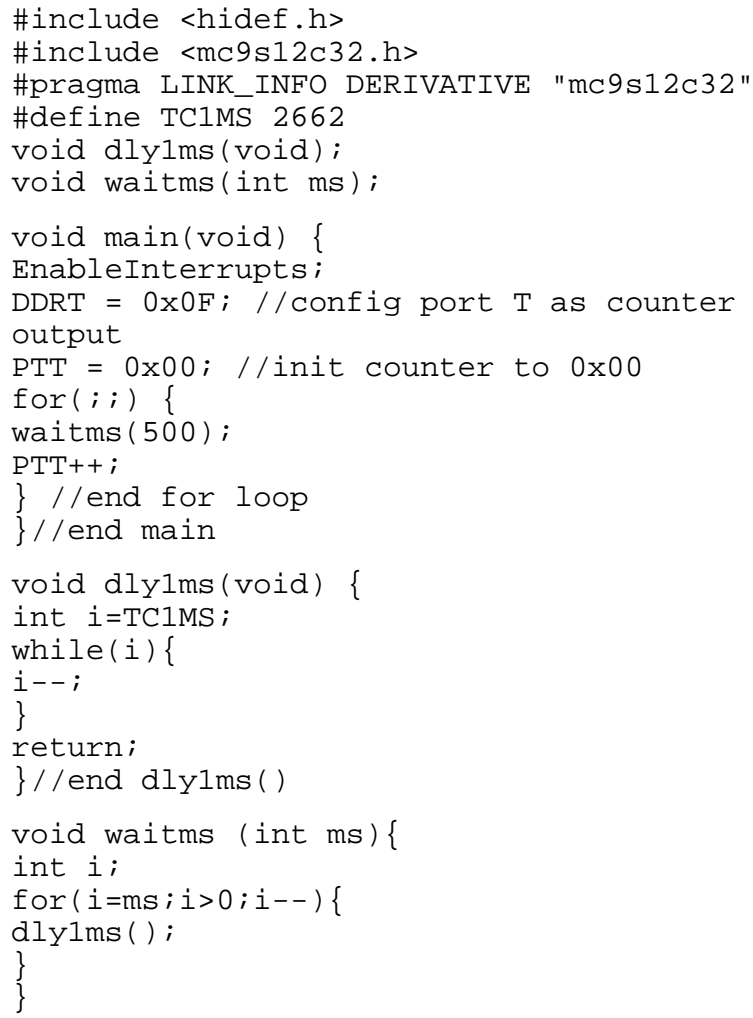

Figure 3. four-bit binary up-counter C program 
The C program was compiled and downloaded into the Freescale MC9S12C32 microcontroller by using the software development tool Freescale CodeWarrior Integrated Development Environment for HCS12 Microcontrollers.

The four-bit up-counter was free running. The four counter signals were available at Port $\mathrm{T}$ of the microcontroller. The Port T outputs were monitored by the digital channels of a Tektronix MSO3012 mixed signal oscilloscope [14]. The four counter signals drove four LEDs for displaying the counter value and were also connected to one end of the logic probe cable. The other end of the logic probe cable was plugged into the digital channels of the mixed signal oscilloscope. The logic probe cable used in the experiments was not a fully shield cable.

The power supply to the Freescale MC9S12C32 microcontroller came from the output of the superposition circuit in Fig. 1. The RF signals used in the experiments were in the range of $7 \mathrm{MHz}$ to $121 \mathrm{MHz}$ in steps of 1 $\mathrm{MHz}$ generated by the HP 8656B Signal Generator. The noisy power supply signal generated by the superposition circuit was monitored by the analog channel of the same Tektronix MSO3012 mixed signal oscilloscope. The Freescale MC9S12C32 microcontroller was integrated into a module in which there were bypass capacitors connecting the microcontroller power pin to ground for filtering out noises.

The main objectives of this lab experiment are described as follows:

- To observe experimentally the effect of noisy power supply on the performance of the counter running on the microcontroller.

- To gain hands on experience for generating radio frequency signals using the HP 8656B Signal Generator.

- To learn how to operate a modern mixed signal oscilloscope for recording analog and digital experimental results simultaneously.

- To introduce the students to the application of the superposition circuit.

- To provide an open-ended research problem for the students to conduct future investigations using the techniques learned in the previous four objectives.

The students performed the experiment and recorded the analog and digital signals simultaneously. They checked whether the counter had any special behavior in the presence of the radio frequency noise on the power supply conductors. They recorded the experimental results and analyzed them.

Their observations are described as follows: the radio frequency generated by the HP 8656B Signal Generator swept was decremented from about $121 \mathrm{MHz}$ to about 7 $\mathrm{MHz}$ with decrement in steps of $1 \mathrm{MHz}$. A typical screen capture of the up-counter waveform on the mixed signal oscilloscope is shown in Fig. 4. The frequency of the RF signal was above $100 \mathrm{MHz}$. There is little or no observable interference occurred in the 4 bit up-counter waveform with noisy power supply. The students expected this because the bypass capacitors should be effective in filtering out the noise in the high end of the frequency range.

When the radio frequency was decreased to about 61 $\mathrm{MHz}$, the students reported that spikes were observed occasionally in the 4 bit up- counter waveform. When the radio frequency signal was decreased further to be below $50 \mathrm{MHz}$, the students reported that more spikes were observed. One of these waveforms was captured by the students and is shown in Fig. 5. A spike is observed at bit 3 of the up- counter waveform. When the radio frequency signal was decreased to about $11 \mathrm{MHz}$, more spikes were observed by the students. One of these waveforms was captured and is shown in Fig. 6.

Students were required to provide concluding remarks based on their observations after this experiment. The first remark was that the operation of the Freescale MC9S12C32 microcontroller was not affected by the emulated electromagnetic interference at the upper range of the frequency band (from about $61 \mathrm{MHz}$ to $120 \mathrm{MHz}$ ). The explanation was that at such high frequency, the corresponding impedance of the bypass capacitors were very small and that became very effective in filtering out the high radio frequency noise.

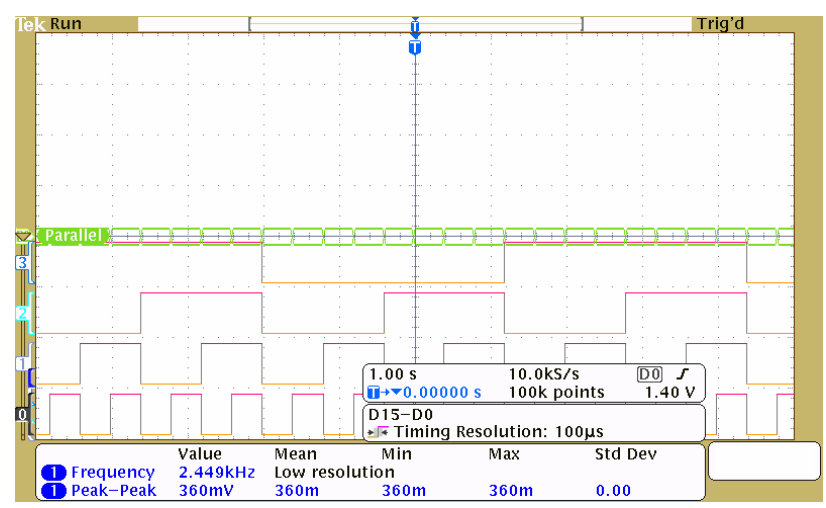

Figure 4. Binary counter waveform without interference

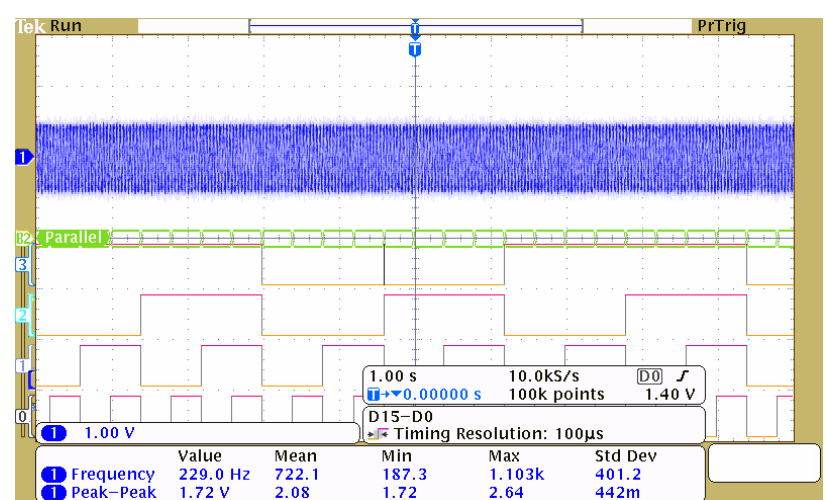

Figure 5. waveform with interference at $21 \mathrm{MHz}$

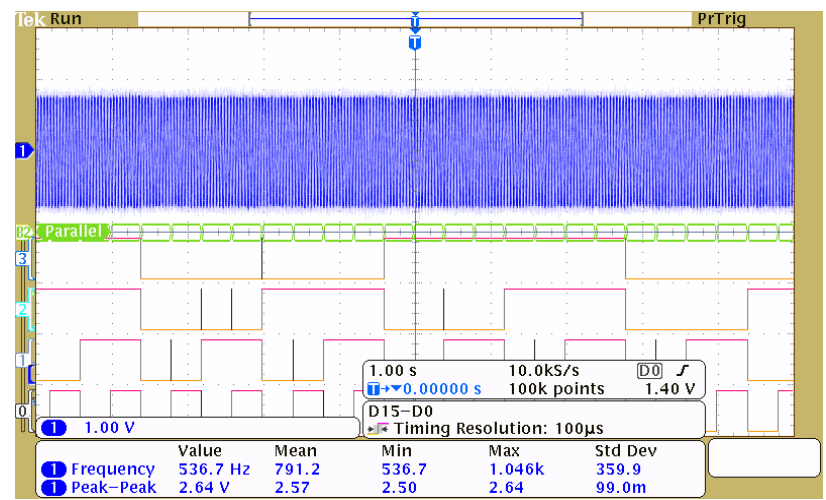

Figure 6. waveform with interference at $11 \mathrm{MHz}$ 
At lower radio frequency of $11 \mathrm{MHz}$ or so, the remark was that the observable spikes were due to the noisy power supply. One of the explanation was that impedance of the bypass capacitors were larger and that became not as effective as before for filtering out the lower radio frequency noise. While this is possible, further explanation was offered to the students. First was that the logic probe cable was not shield fully and was several feet long. The cable itself could pick up lower radio frequency interferences and passed them onto the mixed signal oscilloscope that appeared as spikes. It was suggested that the logic probe cable be shielded fully and to repeat the experiment again so as to investigate whether the spikes can be reduced. Students were encouraged to conduct further research on this open ended problem.

Another explanation of the spikes was that it could be that the spikes were the results of race condition/hazard that could occur in logic gates due to propagation delays of the counter signals. It was necessary to investigate whether the spikes were due to such condition. However, if the cause was due to race condition, one should also observe the spikes initially at the noise frequency of 120 $\mathrm{MHz}$. No spike was observed at such frequency. Therefore the explanation by race condition was ruled out.

\section{B. The second experiment}

Another experiment conducted by the students was the investigation of the effect of possible interference on the pulse width of the binary counter under various RF frequency and power settings. The same Tektronix MSO3012 mixed signal oscilloscope was used to measure the width of the least significant bit of the counter. The RF frequencies injected into the DC source was $88 \mathrm{MHz}, 103 \mathrm{MHz}$, and $118 \mathrm{MHz}$. The power ranged from $0 \mathrm{dBm}$ to $16 \mathrm{dBm}$. The results obtained by the students for the case of $88 \mathrm{dBm}$ are tabulated in Table 1. It was observed that the pulse width did not depend on the RF signal power. The results for the $103 \mathrm{MHz}$ and $118 \mathrm{MHz}$ were the same as the case of the $88 \mathrm{MHz}$.

The objective of this experiment is to provide opportunities for the students to be familiar with the operation and settings of the HP 8656B Signal Generator. Through this lab, the students gained experience of how to choose the power level of the RF signal. A waveform of the

$\mathrm{RF}$ interference signal injected into the $5 \mathrm{~V}$ DC power source is shown in Fig. 7. The second objective of this lab is to learn how to use the mixed oscilloscope for the measurement of pulse width.

The waveform in Fig. 7 also showed the up-counter signal waveform. However, the horizontal time scale was adjusted for observing the RF signal, which frequency was high. Therefore, the up-counter waveform did not show any changes because the counter frequency was very low compared to the RF signal frequency. To observe the state changes of the up-counter waveform, the students increased the horizontal time scale of the oscilloscope.

\section{EVALUATION}

There was much student feedback regarding the electromagnetic interference experiments. First was about the superposition circuit. The circuit was able to combine a DC level and a signal of RF frequency and that was a new concept to the students. They were used to opera tional amplifier summing circuits such as inverting sum-
TABLE I.

PULSE WIDTH OF THE UP-COUNTER

\begin{tabular}{|c|c|}
\hline $\begin{array}{c}\text { RF signal power in dBm } \\
\text { (freq. is 88 MHz) }\end{array}$ & LSB Pulse width (ms) \\
\hline 0 & 399.8 \\
\hline 2 & 399.8 \\
\hline 4 & 399.8 \\
\hline 6 & 399.8 \\
\hline 8 & 399.8 \\
\hline 10 & 399.8 \\
\hline 12 & 399.8 \\
\hline 14 & 399.8 \\
\hline 16 & 399.8 \\
\hline
\end{tabular}

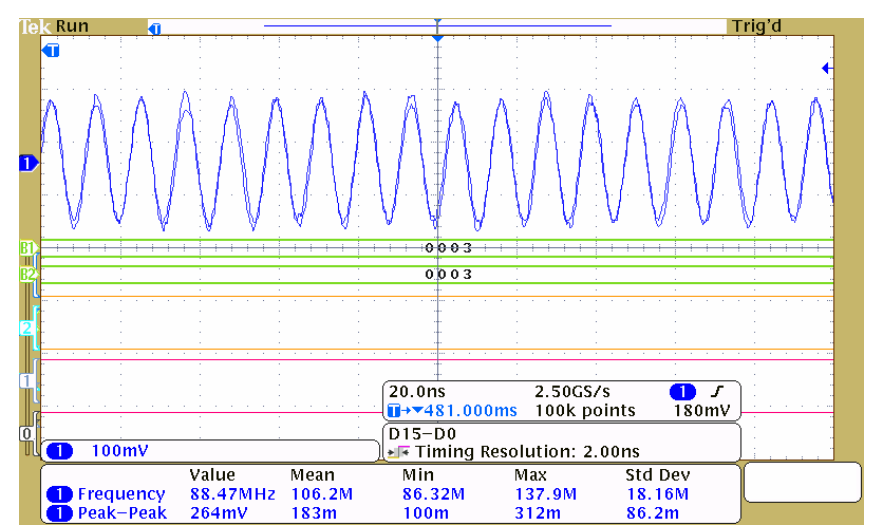

Figure 7. waveform of the RF frequency signal

ming amplifier circuits for combining two signals together. However, such summing circuits are not effective for adding RF frequency signals to a DC source. Through this lab the students learned a new way to combine a DC level and a RF signal through the superposition circuit.

The student also commented on the equipment such as the Tektronix MSO3012 mixed signal oscilloscope and the HP 8656B signal generator. They indicated that they gained valuable hands-on skills through working on these instruments. They admitted that they were a little overwhelmed by these sophisticated instruments in the beginning. However, by following the instructions and the demonstration provided in class, they eventually learned how to operate them. For the mixed signal oscilloscope, the students learned how to configure the digital channels for capturing the up-counter waveforms and the Wave Inspector for analyzing both the up-counter waveform and the RF signal simultaneously. For the HP 8656B signal generator, the students learned how to configure various settings to produce RF signals of different frequencies and power levels.

The open-ended problem of investigating the spikes in the up-counter waveform was not deeply investigated by the students. Some preliminary results obtained by the graduate students of the embedded controls course was that more spikes were observed at higher RF signal frequencies ( $>100 \mathrm{MHz}$ ) when the power level of the RF signal was increased and the capacitance and inductance of the superposition circuit was changed. Further instructions and tutorials will be provided to guide the students in carrying out such investigation.

An up-counter was used in the electromagnetic interference experiments. More sophisticated experiments can be developed in the future, for example, experiments to study the effect of electromagnetic interferences on 
microcontroller on-chip resources such as the analog-todigital converters, serial communication module and the controller area network bus. Electromagnetic interference experiments on these on-chip resources are worth investigating because these on-chip resources are widely used in many applications including wireless applications. The electromagnetic interference can be emulated the same way as in the experiments above by using the superposition circuit, which provides a platform for such investigation in a laboratory environment.

\section{CONCLUDING REMARKS}

Investigating electromagnetic interference on microcontrollers is a practical and relevant topic in embedded systems or electromagnetics courses. This paper describes the lab experiments and the pedagogical approach for covering such topic in those courses. The lab experiments were designed to determine what effect, if any, produced by a noisy power supply upon microcontrollers. The noisy power supply consists of RF signals picked up by a DC voltage that supply power to a microcontroller. The students learned that the RF signals picked up by the conductors of the DC power supply could be emulated by using the superposition circuit. Through observing the up-counter waveform, they realized that at certain frequency of the RF signal, spikes were observed in the upcounter waveform. That became an open-end problem for them to investigate further the cause of this interference.

The pedagogical approach began with well-prepared instructions for conducting the experiments and for using the instruments. It ends with an open-end problem for the students to do further research. The student evaluations were positive as indicated in their lab reports. The students left with experimental skills for this special application at the end of the courses.

\section{REFERENCES}

[1] M. Gilibert, et al., "80C537 Microcontroller Remote Lab for ELearning Teaching," iJOE International Journal of Online Engineering, Vol. 2, No. 4, 2006.
[2] J.W. Bruce; J.C. Harden, and R.B. Reese, "Cooperative and Progressive Design Experience for Embedded Systems," IEEE Transactions on Education, Vol. 47, No. 1, pp. 83- 92, 2004. http://dx.doi.org/10.1109/TE.2003.817618

[3] T.K. Hamrita and R.W. Mcclendon, "A New Approach for Teaching Microcontroller Courses," Int. J. of Engineering Education, Vol. 13, No. 4, pp. 269-274, 1997.

[4] Janos Sztipanovits, et al., "Introducing embedded software and systems education and advanced learning technology in an engineering curriculum," ACM Transactions on Embedded Computing Systems, Vol. 4, No. 3, pp. 549-568, 2005. http://dx.doi.org/ 10.1145/1086519.1086524

[5] A.H.G.Al-Dhaher, "Development of Microcontroller/FPGA-based Systems," International Journal of Electrical Engineering Education, Vol. 20, No. 1, pp. 52-60, 2004.

[6] A.Y. Al-Zoubi, G. Sammour, Q.M. Al-Zoubi, "Utilization of Tablet PCs in Electromagnetics Education,” International Journal of Emerging Technologies in Learning, Vol. 2, No. 2, 2007.

[7] Lars Bengtsson, "Electromagnetic interferences in electrical measurement systems demystified," International Journal of Electrical Engineering Education, Vol. 49, No. 2, pp. 101-113, 2012.

[8] M.F. Iskander, "Technology-based electromagnetic education," IEEE Transactions on Microwave Theory and Techniques, Vol. 50 , Issue 3, pp. 1015-1020, 2002 http://dx.doi.org/10.1109/22.98 $\underline{9985}$

[9] National Highway Transportation Safety Administration, Speedmeasuring Device Performance Specifications: Down-the-road Radar Module, DOT HS 809 812, June 2004.

[10] Hewlett Packard, HP 8656B Signal Generator Operation and Calibration Manual, 1988.

[11] CircuitLab simulation software, https://www.circuitlab.com/

[12] Freescale Semiconductors, Inc., HC9S12C128 datasheet, 2008.

[13] Microchip Technology, Inc., PIC16F631/677/685/687/689/69 Data Sheet (20-Pin Flash-Based, 8-Bit CMOS Microcontrollers with nanoWatt Technology), 2006.

[14] Tektronix MSO3012 mixed signal oscilloscope users manual, 2009.

\section{AUTHOR}

Chiu H. Choi is with University of North Florida, Jacksonville, FL, USA, cchoi@unf.edu

Received 3 Seprtember 2012. Published as resubmitted by the author 18 December 2012. 